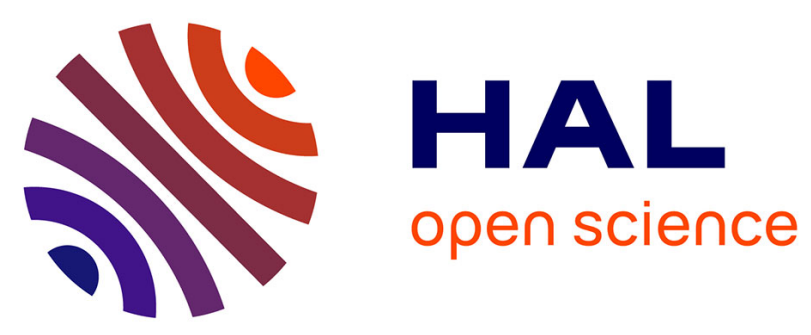

\title{
Automatic spinal cord localization, robust to MRI contrasts using global curve optimization
}

Charley Gros, Benjamin de Leener, Sara Dupont, Allan Martin, Michael

Fehlings, Rohit Bakshi, Subhash Tummala, Vincent Auclair, Donald Mclaren, Virginie Callot, et al.

\section{To cite this version:}

Charley Gros, Benjamin de Leener, Sara Dupont, Allan Martin, Michael Fehlings, et al.. Automatic spinal cord localization, robust to MRI contrasts using global curve optimization. Medical Image Analysis, 2018, 44, pp.215-227. 10.1016/j.media.2017.12.001 . hal-01690881

\section{HAL Id: hal-01690881 https://hal.science/hal-01690881}

Submitted on 11 Jan 2019

HAL is a multi-disciplinary open access archive for the deposit and dissemination of scientific research documents, whether they are published or not. The documents may come from teaching and research institutions in France or abroad, or from public or private research centers.
L'archive ouverte pluridisciplinaire HAL, est destinée au dépôt et à la diffusion de documents scientifiques de niveau recherche, publiés ou non, émanant des établissements d'enseignement et de recherche français ou étrangers, des laboratoires publics ou privés. 


\section{Automatic spinal cord localization, robust to MRI contrasts using global curve optimization}

Charley Gros ${ }^{1}$, Benjamin De Leener ${ }^{1}$, Sara M. Dupont ${ }^{1}$, Allan R. Martin², Michael G. Fehlings $^{2}$, Rohit Bakshi ${ }^{3}$, Subhash Tummala ${ }^{3}$, Vincent Auclair ${ }^{4}$, Donald G. McLaren ${ }^{4}$, Virginie Callot ${ }^{5,6}$, Julien Cohen-Adad ${ }^{1,7}$, Michaël Sdika ${ }^{8}$

1 - NeuroPoly Lab, Institute of Biomedical Engineering, Polytechnique Montreal, Montreal, QC, Canada

2 - Division of Neurosurgery, Department of Surgery, University of Toronto, Toronto, ON, Canada

3 - Laboratory for Neuroimaging Research, Ann Romney Center for Neurologic Diseases, Department of Neurology, Brigham and Women's Hospital, Harvard Medical School, Boston, $M A$

4 - Biospective Inc., Montreal, QC, Canada

5 - Aix Marseille Univ, CNRS, CRMBM, Marseille, France

6 - APHM, Hôpital de la Timone, Pôle d'imagerie médicale, CEMEREM, Marseille, France

7 - Functional Neuroimaging Unit, CRIUGM, Université de Montréal, Montreal, QC, Canada

8 - Univ.Lyon, INSA-Lyon, Université Claude Bernard Lyon 1, UJM-Saint Etienne, CNRS, Inserm, CREATIS UMR 5220, U1206, F-69100, LYON, France

\section{DECLARATION OF INTEREST}

Donald G. McLaren and Vincent Auclair are currently employees of Biospective, Inc.

\section{Corresponding Author:}

Michaël Sdika

CREATIS - INSA LYON

Bâtiment Blaise Pascal

7 Avenue Jean Capelle

69621 Villeurbanne Cedex

FRANCE

skype: msdika e-mail: $\underline{\text { michael.sdika@creatis.insa-lyon.fr }}$

\section{Abstract}

During the last two decades, MRI has been increasingly used for providing valuable quantitative information about spinal cord morphometry, such as quantification of the spinal 
cord atrophy in various diseases. However, despite the significant improvement of MR sequences adapted to the spinal cord, automatic image processing tools for spinal cord MRI data are not yet as developed as for the brain. There is nonetheless great interest in fully automatic and fast processing methods to be able to propose quantitative analysis pipelines on large datasets without user bias. The first step of most of these analysis pipelines is to detect the spinal cord, which is challenging to achieve automatically across the broad range of MRI contrasts, field of view, resolutions and pathologies. In this paper, a fully automated, robust and fast method for detecting the spinal cord centerline on MRI volumes is introduced.

The algorithm uses a global optimization scheme that attempts to strike a balance between a probabilistic localization map of the spinal cord center point and the overall spatial consistency of the spinal cord centerline (i.e. the rostro-caudal continuity of the spinal cord). Additionally, a new post-processing feature, which aims to automatically split brain and spine regions is introduced, to be able to detect a consistent spinal cord centerline, independently from the field of view. We present data on the validation of the proposed algorithm, known as “OptiC", from a large dataset involving 20 centers, 4 contrasts $\left(T_{2}\right.$-weighted $n=287, T_{1}$ weighted $n=120, T_{2}{ }^{*}$-weighted $n=307$, diffusion-weighted $n=90$ ), 501 subjects including 173 patients with a variety of neurologic diseases. Validation involved the gold-standard centerline coverage, the mean square error between the true and predicted centerlines and the ability to accurately separate brain and spine regions.

Overall, OptiC was able to cover $98.77 \%$ of the gold-standard centerline, with a mean square error of $1.02 \mathrm{~mm}$. OptiC achieved superior results compared to a state-of-the-art spinal cord localization technique based on the Hough transform, especially on pathological cases with an averaged mean square error of $1.08 \mathrm{~mm}$ vs. $13.16 \mathrm{~mm}$ (Wilcoxon signed-rank test pvalue $<0.01$ ). Images containing brain regions were identified with a $99 \%$ precision, on which brain and spine regions were separated with a distance error of $9.37 \mathrm{~mm}$ compared to groundtruth.

Validation results on a challenging dataset suggest that OptiC could reliably be used for subsequent quantitative analyses tasks, opening the door to more robust analysis on pathological cases.

Keywords: Spinal cord, MRI, Detection, Segmentation, Global Optimization, Machine learning

Abbreviations:

SC: Spinal Cord

PMJ: Pontomedullary junction

FOV: Field of View

(S-I) axis: Superior-Inferior axis

HOG: Histogram of Oriented Gradient

SVM: Support Vector Machine

MS: Multiple Sclerosis

DCM: Degenerative Cervical Myelopathy

NMO: Neuromyelitis Optica

SCI: Spinal Cord Injury

ALS: Amyotrophic Lateral Sclerosis

SYR: Syringomyelia

MSE: Mean Square Error

HC: Healthy Controls

\section{Introduction}

The spinal cord (SC) plays a key role in the central nervous system by ensuring the conduction of both motor and sensory signaling between the brain and the peripheral nervous systems. Although SC magnetic resonance imaging (MRI) has long been technically challenging, MRI has been increasingly used in the last two decades to provide valuable quantitative information through SC morphometry (Fradet et al., 2014; Martin et al., 2017b; Papinutto et al., 2015) and to evaluate SC damage a range of neurologic disorders such as multiple sclerosis, traumatic, and neurodegenerative diseases (Cawley et al., 2017; CohenAdad et al., 2013, 2011; Ellingson et al., 2008; Grabher et al., 2017; Martin et al., 2017a; 
Nakamura et al., 2008). With newer acquisition techniques that enhance the quality of SC MRI data (Stroman et al., 2014), there is a need for improved image processing methods to propose robust automated quantitative analysis pipelines.

Localizing the SC on MRI scans is a key step for automating quantitative analysis pipelines such as SC (De Leener et al., 2014; Horsfield et al., 2010) and grey matter (Dupont et al., 2017; Prados et al., 2017, 2016) segmentations, template registration (De Leener et al., 2017b; Stroman et al., 2008) and B0 susceptibility-related distortion correction (Johanna Vannesjo et al., 2017; Topfer et al., 2016). While localizing the SC might appear as a rudimentary computerized task, it is much more challenging to achieve it robustly and accurately across a broad range of SC shapes, craniocaudal vertebral length, pathologies, image field of view (FOV), image resolution and orientation, types of contrast and image artifacts (e.g. susceptibility, motion, chemical shift, ghosting, blurring, Gibbs). For instance, about 20 recently-published SC segmentation methods still require manual intervention for initialization and optimization (De Leener et al., 2016), especially to identify specific anatomic landmarks within the SC. Hence, SC segmentation methods could be made fully automatic if initialized with a robust automated SC centerline localization module.

Most of the existing automatic SC localization methods took advantage of the ellipsoid shape of the SC in cross-section and are based on the Hough transform with vesselness filtering (De Leener et al., 2014), active contour (Koh et al., 2010), or continuous max-flow with cross-sectional similarity prior (Pezold et al., 2015). Other methods are based on energy-minimization methods through image-based strategies, by pattern registration with partial volume effect modelization (Carbonell-Caballero et al., 2006) or atlas registration with topology constraint (Chen et al., 2013). While these automatic algorithms have shown good performance (De Leener et al., 2016), they often require a specific region of interest or are limited to specific contrast and resolution.

This paper introduces a novel fully automatic, accurate and fast algorithm (OptiC) to detect the center of the SC. OptiC is based on a supervised machine learning detection method combined with a distance-transform-based global optimization. Its performance was evaluated on non-processed data (i.e., not cropped, filtered, reoriented, intensity-normalized, etc.) across a large multi-center dataset from 20 sites $(n=20)$ including a variety of image quality, resolution and pathologies across four image contrasts: $T_{2}$-weighted $\left(T_{2} W, n=287\right), T_{1}$ weighted $\left(T_{1} W, n=120\right), T_{2}{ }^{*}$-weighted $\left(T_{2}{ }^{*} \mathrm{w}, \mathrm{n}=307\right)$ and diffusion weighted (DWI, $\left.n=90\right) 3 \mathrm{D}$ images. For the sake of transparency and reproducibility, we have made this method publicly available as part of the Spinal Cord Toolbox ${ }^{1}$ (v3.0.2 and above) free and open-source software (De Leener et al., 2017a) and on the webpage of the CREATIS lab².

The main contributions of this work are (i) an original framework to robustly find the SC centerline, based on machine learning and global curve optimization, (ii) an efficient algorithm to solve the optimization problem in a non-iterative way with linear complexity, (iii) a robust ponto-medullary junction (PMJ) detector to separate brain and spine regions and (iv) an extensive validation on a broad dataset involving several centers, pathologies, MR contrasts and image resolutions.

In the following sections, the theoretical aspects of OptiC will be detailed, along with the optimization of its hyperparameters and the evaluation across the testing dataset. Second, the results of the training and testing steps will be presented. Finally, the key contributions of OptiC method will be discussed.

1 sourceforge.net/projects/spinalcordtoolbox/

2 creatis.insa-lyon.fr/site7/en/ctrDetect 


\section{Material and Methods}

\subsection{Theory}

In the following subsections, the OptiC approach will be detailed and a new algorithm will be proposed to efficiently find a global minimum to the optimization problem. The final section presents an automatic post-processing method to differentiate brain and spinal cord regions in order to prevent analysis of the brain region.

\subsubsection{OptiC SC centerline detection}

The OptiC method relies on two prior anatomical sets of knowledge: (i) the SC has a specific tubular shape and (ii) the SC centerline is continuous along the superior-inferior (S-I) (cranio-caudal) axis. Consequently, the OptiC approach encodes these priors in 2 distinct steps (see Figure 1): (i) step 1: a SC localization map is computed, using standard object detection tools, (ii) step 2: an optimization problem is solved to take into account the global tubular shape of the SC. As a result, the SC centerline is modeled as a curve compromising the tubular similarity and the curve continuity. 


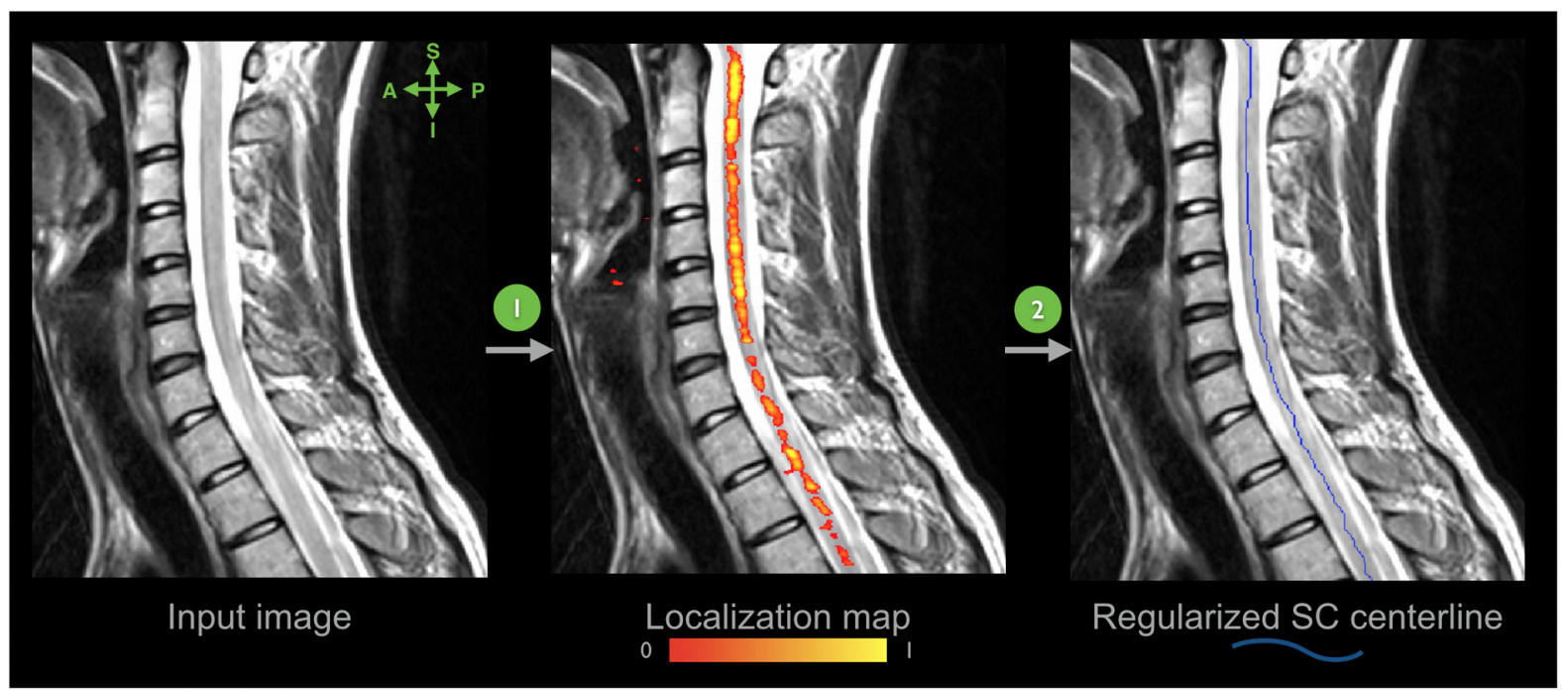

Figure 1. OptiC pipeline overview. OptiC is based on two steps: (1) The spinal cord (SC) probabilistic localization map is computed from the input image, (2) The SC centerline is modeled as a regularized curve, constrained by a trade-off between the localization map values and the SC continuity in the superior-to-inferior (S-I) axis.

\subsubsection{Step1: Spinal cord localization map}

The goal of the first step is to compute a localization map of the SC, in a supervised manner, based on the detection of its specific ellipsoidal shape. It is achieved by predicting the presence of the SC in a given 2D image-patch with a classification model, as commonly done for object detection. Trained on labeled image-patches, the classification model assigns to each voxel a confidence of SC presence within the corresponding patch, resulting in a SC localization map.

\subsubsection{Step2: Spinal cord centerline modeling}

From the previously computed SC localization map, the goal of the second step is to model the SC as a curve which constrains the centerline continuity. Thus, the resulting centerline $C$ is a regularized curve compromising the localization map $S$ values and the SC continuity, which could be expressed as the solution of the following optimization problem (Eq. 1):

$$
\min _{c_{0}, \ldots, c_{n-1}} C_{n-1}\left(c_{0}, \ldots, c_{n-1}\right)=-\sum_{z=0}^{n-1} S_{z}\left(c_{z}\right)+\lambda \sum_{z=0}^{n-2}\left\|c_{z+1}-c_{z}\right\| \square^{2}
$$

with $\lambda$, a regularization coefficient; $C_{z}$, the SC center and $S_{z}$, the localization map on the $\mathrm{z}^{\text {th }}$ slice; $n$, the number of slice in the 3D input MRI volume. Thus, the optimization problem has to make a trade-off between the classifier confidence in the SC localization, and the SC continuity between adjacent slices, respectively encoded by the first and second terms of (Eq 1). 


\subsubsection{Optimization problem resolution}

The optimization problem (Eq. 1) could be solved using a gradient descent approach. This would be efficient but would not ensure finding the global solution. A brute force algorithm would find the global solution but would be impractical with a $O\left(s^{n}\right)$ complexity, where $s$ is the number of voxels per slice and $n$ the number of slices in the 3D MRI volume).

In this section, a novel algorithm is detailed to efficiently solve (Eq. 1) and guarantee robustness of the optimization problem since it provides a global solution. Based on distancetransforms, this approach has a complexity linear with the number of voxels, in $O(s . n)$.

To solve the problem, an auxiliary sequence of 2D images $\left(M_{k}\right)$ is defined as:

$$
M_{k}\left(x_{k}\right)=\min _{x_{0}, \ldots, x_{k-1}} C_{k}\left(x_{0}, \ldots, x_{k}\right) .
$$

with $k$ the slice number. Slices above the index $k$ are removed, and the problem is seen as a function of the center on slice $k$ only. If the $M_{n-1}$ image is known, the relation

$$
\min _{x_{n-1}} M_{n-1}\left(x_{n-1}\right)=\min _{x_{0}, \ldots, x_{n-1}} C_{n-1}\left(x_{0}, \ldots, x_{n-1}\right) \text {, }
$$

enables finding the center solution on the last slice:

$$
c_{n-1}=\arg \min _{x_{n-1}} M_{n-1}\left(x_{n-1}\right) \text {. }
$$

However, computing the $M_{n-1}$ image is as difficult as solving the original problem. Fortunately, the sequence $\left(M_{k}\right)$ satisfies the following recurrence relationship:

$$
\begin{aligned}
M_{k}\left(x_{k}\right) & =\min _{x_{0}, \ldots, x_{k-1}}-\sum_{z=0}^{k} S_{z}\left(x_{z}\right)+\lambda \sum_{z=0}^{k-1}\left\|x_{z+1}-x_{z}\right\| \square^{2} \\
& i-S_{k}\left(x_{k}\right)+\lambda \min _{x_{k-1}}\left\{\frac{M_{k-1}\left(x_{k-1}\right)}{\lambda}+\left\|x_{k}-x_{k-1}\right\| \square^{2}\right\} \\
& i-S_{k}\left(x_{k}\right)+\lambda D\left(\frac{M_{k-1}}{\lambda}\right)\left(x_{k}\right)
\end{aligned}
$$

with $D$ defined by the image operator $D(f)(x)=\min _{y} f(y)+\|x-y\| \square^{2}$. This recurrence relation not only enables us to practically compute the $\left(M_{k}\right)$, it also provides a way to infer the full centerline, solution of (Eq. 1), from the top to the bottom. Indeed, given $c_{k}$ , $\quad C_{k-1}$ can be expressed as:

$$
c_{k-1}=\operatorname{argmin}_{x_{k-1}}\left\{\frac{M_{k-1}\left(x_{k-1}\right)}{\lambda}+\left\|c_{k}-x_{k-1}\right\| \square^{2}\right\}
$$




$$
i N\left(\frac{M_{k-1}}{\lambda}\right)\left(c_{k}\right)
$$

(Eq. 4)

where $\quad N$ is the proximity operator defined as $N(f)(x)=\operatorname{argmin}_{y}\left\{f(y)+\|x-y\| \square^{2}\right\}$. $D$ and $N$ are important operators for convex optimization and image processing. They can be used to compute the distance transform and the nearest neighbors transform of an image. As proposed previously (Felzenszwalb and Huttenlocher, 2004; Meijster et al., 2002), these two transforms can be computed efficiently (with linear complexity) when the input function is uniformly sampled, i.e. is an image.

The resolution of (Eq. 1) is formalized in Algorithm 1. Fast distance and nearestneighbor transforms are first used to compute the sequences $\left(M_{k}\right)$ and $\left(N_{k}\right)$ from the bottom to the top of the volume. On the top slice, the SC center is then found as the minimum of $M_{n-1}$. The complete centerline sequence $(\stackrel{c}{i \dot{i} k)}$ is then inferred by back-tracking on i

the nearest-neighbor-transforms $\left(N_{k}\right)$. As the computation of $M_{k}$ and $N_{k}$ is linear, in $O(s)$, the complexity of OptiC is also linear, in $O(s . n)$.

The proof that the Algorithm 1 finds a global solution of the problem defined by (Eq. 1) is fairly simple. Using (Eq. 3), the minimal value of $C_{n-1}, \widehat{C_{n-1}}$, is given by the global minimization of $M_{n-1}$. This global minimization is performed by looping on the pixel of the $M_{n-1}$ 2D image. The backtracking procedure given by (Eq. 4) ensures finding a path, a $\left.\begin{array}{c}c \\ (\dot{i} \dot{i} k)\end{array}\right)$ sequence, satisfying $C_{n-1}\left(c_{0}, \ldots, c_{n-1}\right)=\widehat{C_{n-1}}$ and thus it enables finding a global solution of the optimization problem.

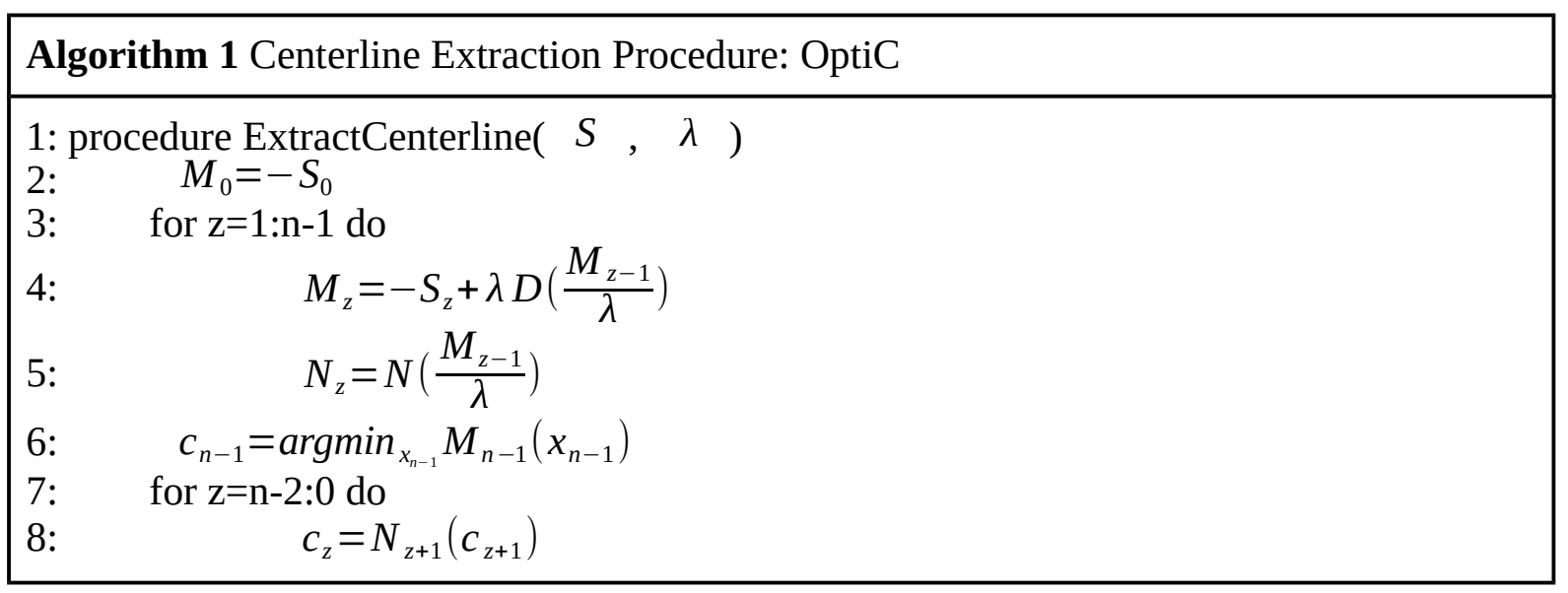

\subsubsection{Implementation details}

In the optimization problem, the $z$ coordinate was assumed to be the (S-I) axis for all input volumes. Thus, for each MRI volume, preprocessing steps involved imposing a common image orientation (RPI, i.e. Right-to-left, Posterior-to-anterior, Inferior-to-superior) based on the image header (NIFTI input).

In order to be robust to the native image resolution, the input image is re-interpolated 
to the resolution $1 \times 1 \times p_{z}$ mm using trilinear interpolation, $p_{z}$ being the native pixel size in $z$ direction. To account for the image resolution in the (S-I) axis, the regularization coefficient $\lambda$ is parameterized with a resolution independent parameter $\lambda^{\prime}$ and the interslice distance $p_{z}$ :

$$
\lambda=\frac{\lambda^{\prime}}{p_{z}^{2}}
$$

The SC localization map was computed using a Support Vector Machine (SVM) trained with Histogram of Oriented Gradient features (HOG) as described previously (Dalal and Triggs, 2005). The HOG features were computed on patches of $32 \times 32$ size, from $2 \mathrm{D}$ axial slices, using the OpenCV library (Bradski and Kaehler, 2008) with default parameters and signed gradients. Note that the use of a small patch size as well as the sophisticated normalization scheme of HOG makes the computation of the localization map robust to inhomogeneity artifacts and intensity scale changes. 


\subsubsection{Pontomedullary junction detection}

Typical cervical SC scans include a part (or all) of the brain, which therefore needs to be excluded in the SC centerline output of the proposed algorithm. Here, an automatic method is introduced to detect the presence and position of the PMJ and subsequently crop the SC centerline above it. This method is included in OptiC as a post-processing module. The choice of the PMJ is driven by previous studies showing that this anatomical landmark could be used as a reference for SC template-based analyses (Stroman et al., 2008). Moreover, this anatomical landmark can be precisely identified from multiple image contrasts and its neighboring voxels exhibit appropriate features for machine learning methods.

Figure 2 details the post-processing pipeline to detect the PMJ. The SC is first straightened along the extracted centerline, with a robust algorithm that preserves the SC topology and its internal and adjacent structure (De Leener et al., 2017b). Straightening of the SC is used to normalize the shape of the PMJ regardless of the patient position during the MRI scan. To detect the presence of the PMJ on the straightened volumes, a HOG+SVM 2D classifier (as described in section 2.1.1.4.) is trained on the midsagittal slice of the straightened training data, resampled to a $1 \times 1 \mathrm{~mm}$ resolution. When the PMJ is detected on the testing data, the centerline is cropped above it.

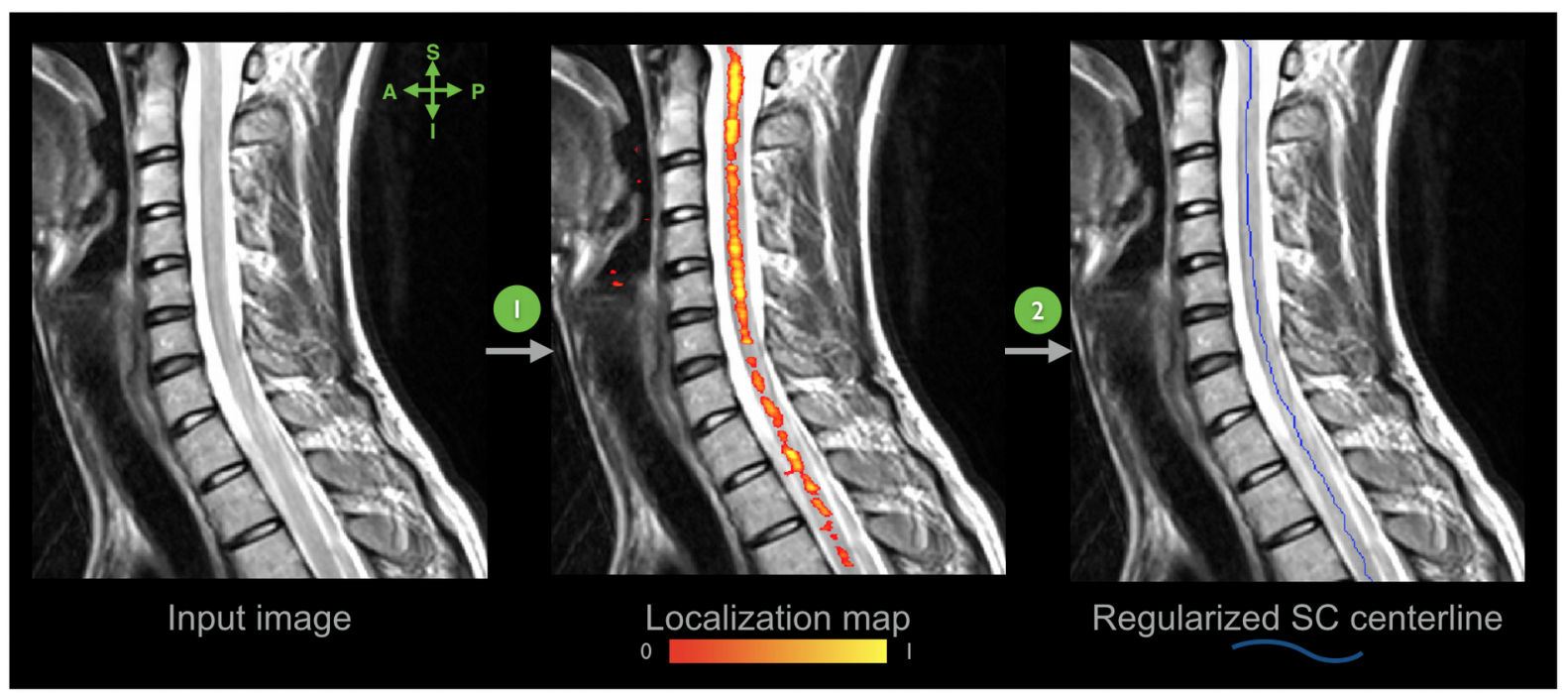

Figure 2. Automatic post-processing pipeline to restrict the OptiC centerline below the ponto-medullary junction (PMJ). The spinal cord (SC) is first straightened along the regularized SC centerline (blue). A classifier localization map (heat probabilistic map) allows detection of the PMJ on the straightened image (middle). Finally, the SC centerline is restricted below the detected PMJ (right). 


\subsection{Materials}

To make sure the algorithm performs well in a large variety of data (i.e., robust to MRI vendor, contrast, sequence parameters, artifacts, types of pathology, etc.), retrospective data from 20 research and clinical centers were collected. The dataset was composed of 501 subjects, including 173 patients with neurologic conditions [multiple sclerosis (MS) $n=79$, degenerative cervical myelopathy $(\mathrm{DCM}) \mathrm{n}=63$, neuromyelitis optica $(\mathrm{NMO}) \mathrm{n}=19$, traumatic spinal cord injury (SCI) $n=5$, amyotrophic lateral sclerosis (ALS) $n=5$, syringomyelia (SYR) $\mathrm{n}=2$ ]. Volumes were acquired on 3T systems using standard coils, spanning a broad range of FOVs (e.g. cervical, thoracic, including or not brain sections) and four different contrasts: $\mathrm{T}_{2} \mathrm{~W}(\mathrm{n}=287), \mathrm{T}_{1} \mathrm{~W}(\mathrm{n}=120), \mathrm{T}_{2}{ }^{*} \mathrm{~W}(\mathrm{n}=307)$ and DWI $(\mathrm{n}=90)$. Spatial resolutions included isotropic ( $\mathrm{n}=366$, from 0.7 to $1.3 \mathrm{~mm})$ and anisotropic data with axial $(\mathrm{n}=430$, in plane: from 0.3 to $1.5 \mathrm{~mm}$, slice thickness: from 1.0 to $24.5 \mathrm{~mm}$ ), or sagittal orientation ( $\mathrm{n}=8$, in plane: from 0.4 to $0.7 \mathrm{~mm}$, slice thickness: from 0.8 to $2.8 \mathrm{~mm}$ ). Demography and resolution are summarized in Figure 3. 


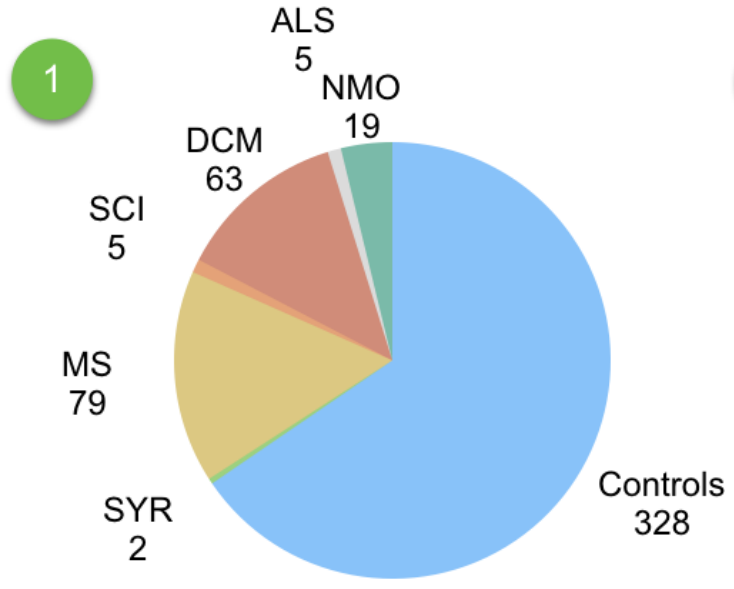

501 subjects from 20 centers.

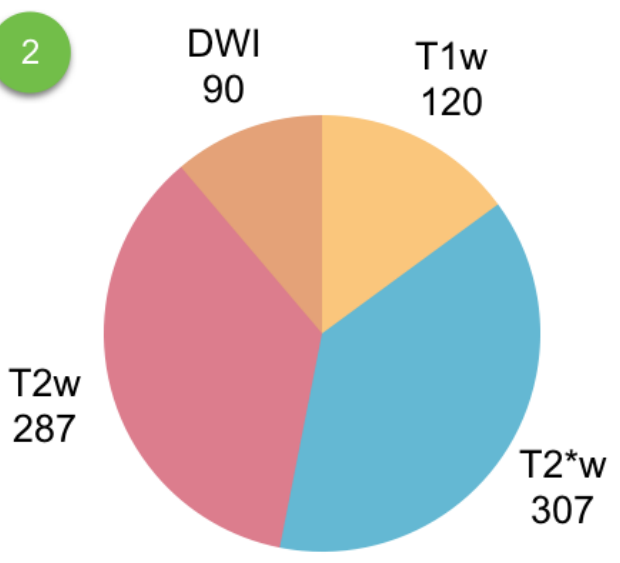

804 volumes from 4 contrasts.

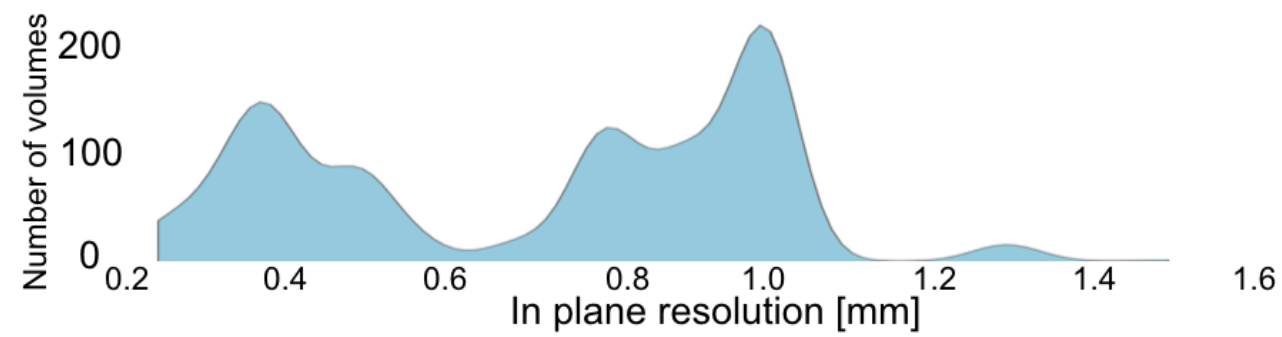

$3 \mathrm{~b}$

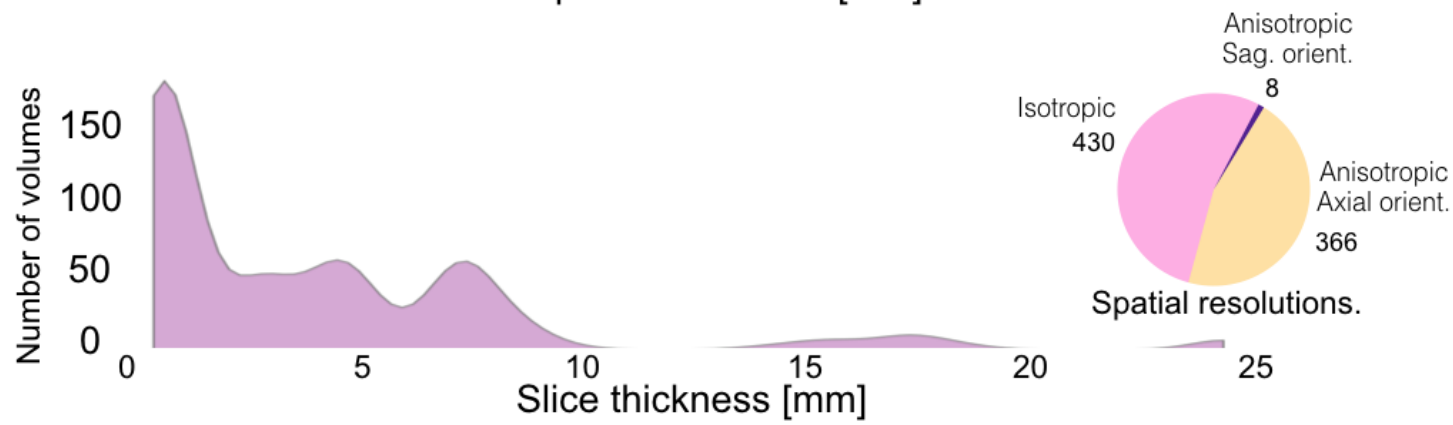

Figure 3. Demography and resolution. Retrospective data from a large variety of pathologies (1) and contrasts (2) were used. For clarity purposes, the distribution of subjects is only shown for pathologies and contrasts. Volumes were acquired at 3T, spanning a large range of slice orientations, in plane resolutions (3a) and slice thickness (3b). Abbreviations: MS: multiple sclerosis; DCM: degenerative cervical myelopathy; NMO: neuromyelitis optica; SCI: Traumatic spinal cord injury; ALS: amyotrophic lateral sclerosis; SYR: syringomyelia.

For each of the four contrasts, the dataset $\left(\mathrm{N}_{\text {tot }}\right.$ volumes) was randomly split into two independent subsets, the validation ( $\mathrm{N}_{\text {valid }}$ volumes) and the testing $\left(\mathrm{N}_{\text {test }}\right.$ volumes) datasets, as illustrated in Figure 4. The training-validation dataset (used to train and validate the model) involved 40 healthy controls (HC), and the testing dataset involved the rest of the HC, and 
patients. The reason for not including patient data for the training-validation was for providing an unbiased and generic model, which would then be tested on patients (ultimately, this algorithm could be applied for diagnosis purposes, hence without prior knowledge of the possible pathology). The center of the SC was manually localized using FSLView (Jenkinson et al., 2012) on each slice of each volume as ground truth for the SC centerline. Similarly, the PMJ position was manually localized on the midsagittal slice of each straightened $T_{1} \mathrm{~W}$ and $\mathrm{T}_{2} \mathrm{~W}$ volume with brainstem section.

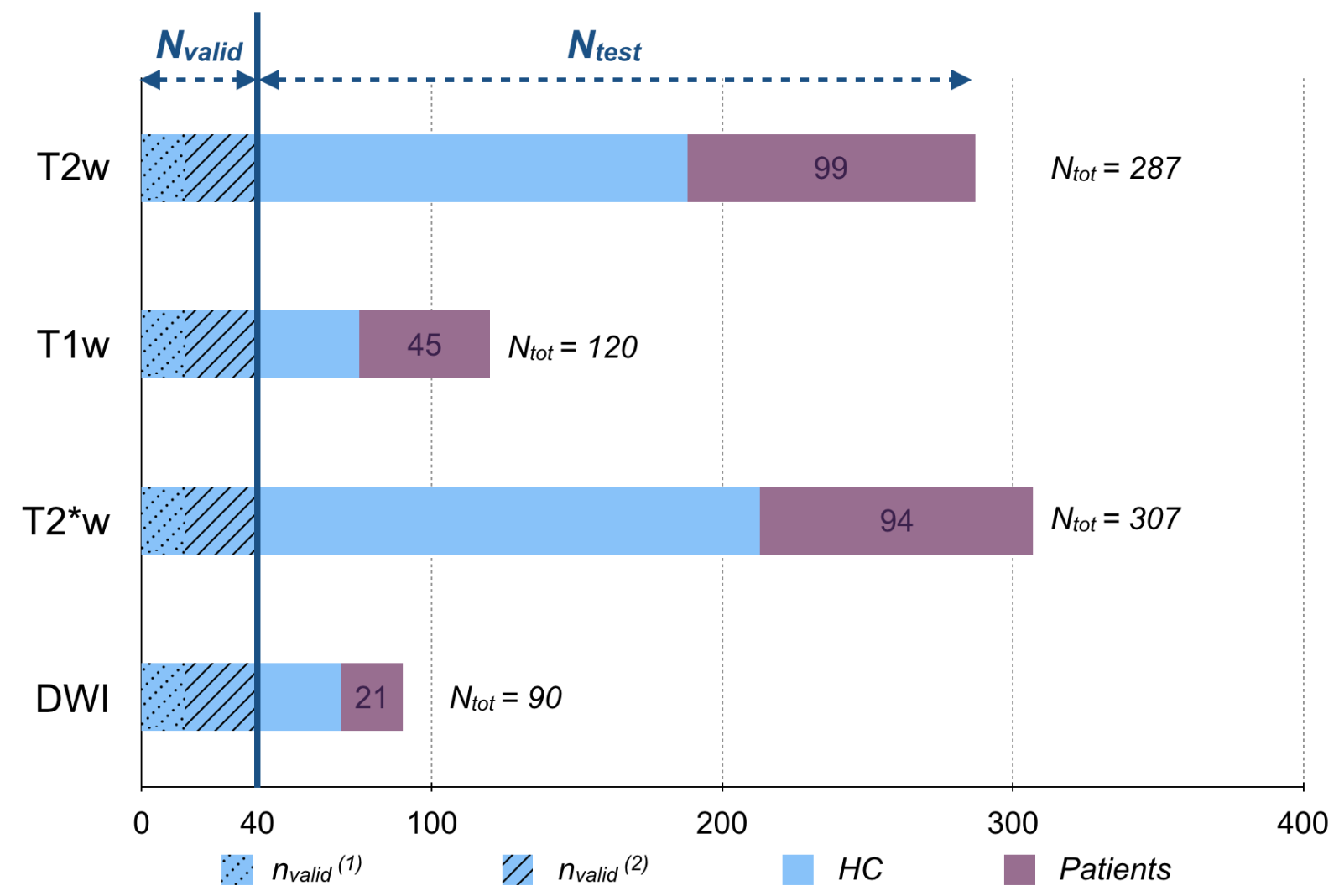

Figure 4. Training-validation and testing dataset breakdown. The dataset of each contrast $\left(\mathrm{N}_{\text {tot }}\right)$ was randomly split into two independent subsets: the training-validation $\left(\mathrm{N}_{\text {valid }}=40\right)$ and the testing $\left(\mathrm{N}_{\text {test }}=\mathrm{N}_{\text {tot }}-\mathrm{N}_{\text {valid }}\right)$ datasets. The training-validation dataset only involved healthy controls (HC, in blue) whereas the testing dataset involved both patients (in purple) and HC. The training-validation data were also split into 2 independent subsets: the first $\left(\mathrm{n}_{\text {valid }}{ }^{(1)}\right)$ for model training, the second $\left(\mathrm{n}_{\text {valid }}{ }^{(2)}\right)$ for evaluation.

\subsection{Hyperparameters optimization}

Three hyperparameters were investigated: the number of training volumes, the effect of rotation augmentation in training, and the regularization coefficient $\lambda^{\prime}$. To do so, for each hyperparameter, 100 bootstrap iterations were run on the training-validation dataset $\left(\mathrm{N}_{\text {valid }}\right)$, split into two independent data subsets: one for training $\left(\mathrm{n}_{\text {valid }}{ }^{(1)}\right.$ from which the training volumes were selected), the other for evaluation $\left(\mathrm{n}_{\text {valid }}{ }^{(2)}\right.$, common for all the tested values of the current hyperparameter), as detailed in Figure 4. The best model was selected by 
computing the Mean Square Error (MSE) between the predicted $\widehat{C}$ and the ground-truth $C$ centerlines:

$$
M S E=\sqrt{\square}
$$

with $n$ is the number of $2 \mathrm{D}$ axial slices. For each hyperparameter, significant differences between the tested values were investigated with a Kruskal-Wallis H-test. After optimization, each hyperparameter was fixed for the remainder of the study.

\subsubsection{Number of training images}

In this part, we investigated if increasing the number of 3D images used to train the model yielded a more robust SC detection. Based on metrics measured on a validation dataset, models with the following number of training volumes were compared: $1,5,10,15$. The number of training 3D images $\left(n_{\text {train }}\right)$ achieving the best results were retained for the rest of the study (i.e. $n_{\text {valid }}^{(1)}=n_{\text {train }}$ and $n_{\text {valid }}^{(2)}=N_{\text {valid }}-n_{\text {train }}$ ).

\subsubsection{Rotation training augmentation}

Data augmentation is a common strategy to increase detection robustness in machine learning. This study investigated whether the model can be made rotation invariant or whether adding a rotation to the input volume improved the model generalization and prevented overfitting. Rotations around the (S-I) axis were applied to the training data $\left(\mathrm{n}_{\text {train }}\right)$ with different angular ranges $\left( \pm 30^{\circ}\right.$ and $\pm 180^{\circ}$ ) and angular increments (each $5^{\circ}$ and each $\left.10^{\circ}\right)$.

\subsubsection{Regularization coefficient}

The regularization coefficient $\lambda^{\prime}$ is a hyperparameter that affects the (S-I) axis regularization of the extracted centerline. The higher $\lambda^{\prime}$ is, the more the centerline points from two adjacent slices tend to converge in the X-Y plane. To optimize $\lambda^{\prime}$, discrete values were investigated from 0 to 4 . Note that, when $\lambda^{\prime}$ is null, the centerline is given by the maximal values of the SC localization map, and when $\lambda^{\prime}$ goes to infinity, the centerline will tend to be a vertical straight line.

\subsection{Evaluation}

Once the OptiC hyperparameters were selected, OptiC performance was evaluated on the testing dataset $\left(\mathrm{N}_{\text {test }}\right.$ ), using both $\mathrm{HC}$ and patients (see Figure 4).

\subsubsection{OptiC SC centerline detection}

For each contrast, an OptiC optimized model was evaluated on the testing dataset and compared to a recently-published automatic SC localization method (De Leener et al., 2015), based on the Hough transform detection with vesselness filtering (referred as Hough in the following paragraphs). Validation metrics were MSE between the predicted and ground truth SC centerlines and the localization rate, defined as the percentage of slices for which the predicted centerline was included in the manually-segmented SC. 


\subsubsection{Pontomedullary junction detection}

To achieve fully automatic pipelines involving multi-center datasets with a variety of FOVs, the PMJ detection is a mandatory post-processing step to restrict the centerline curve to SC regions. The proposed method to detect the PMJ was evaluated on $\mathrm{T}_{2} \mathrm{~W}$ and $\mathrm{T}_{1} \mathrm{~W}$ datasets that included a large number of volumes with brain sections: PMJ appeared on 45\% of $\mathrm{T}_{2} \mathrm{~W}$ and $90 \%$ of $\mathrm{T}_{1} \mathrm{~W}$ volumes of the testing dataset. The training and testing datasets were the same as in the previous section.

The detection performance was assessed with recall (measure of completeness) and precision (measure of exactness) metrics:

$$
\begin{gathered}
\text { recall }=\frac{T P}{T P+F N} \\
\text { precision }=\frac{T P}{T P+F P}
\end{gathered}
$$

where TP (true positives) and FN (false negatives) are the number of volumes with brain section where the PMJ is respectively detected and not detected; FP (false positives) are the number of volumes on which PMJ is detected even though the brain section is not present.

For volumes with detected PMJ, the localization accuracy is evaluated by computing the distance between the predicted and true PMJ position along the (S-I) axis.

\subsubsection{Proof-of-concept: application to SC segmentation}

SC segmentation provides relevant morphometric information about SC atrophy (Yiannakas et al., 2016) and is an important step in template-based analysis pipelines (De Leener et al., 2017a). Thus, as a proof-of-concept application, a SC segmentation method PropSeg (De Leener et al., 2015), which uses propagation of 3D meshes, was initialized using an OptiC centerline to compare its benefit over the default initialization. Dice coefficient (Dice, 1945), between the predicted and ground-truth segmentations (done by experienced users), evaluated the quality of the segmentation of the testing subjects. The computation time was also measured for the SC segmentation process when initialized with OptiC versus with the default version. 


\section{Results}

Figure 5 presents the results of the hyperparameter optimization for each contrast. The selected hyperparameters were chosen through a bootstrap analysis, as a trade-off between detection accuracy (measured by the MSE) and simplicity of the model. The OptiC hyperparameter (filled in green) was the same for all contrast, selected as either the best tested value or not significantly different from the best tested value, according to KruskalWallis H-tests. 

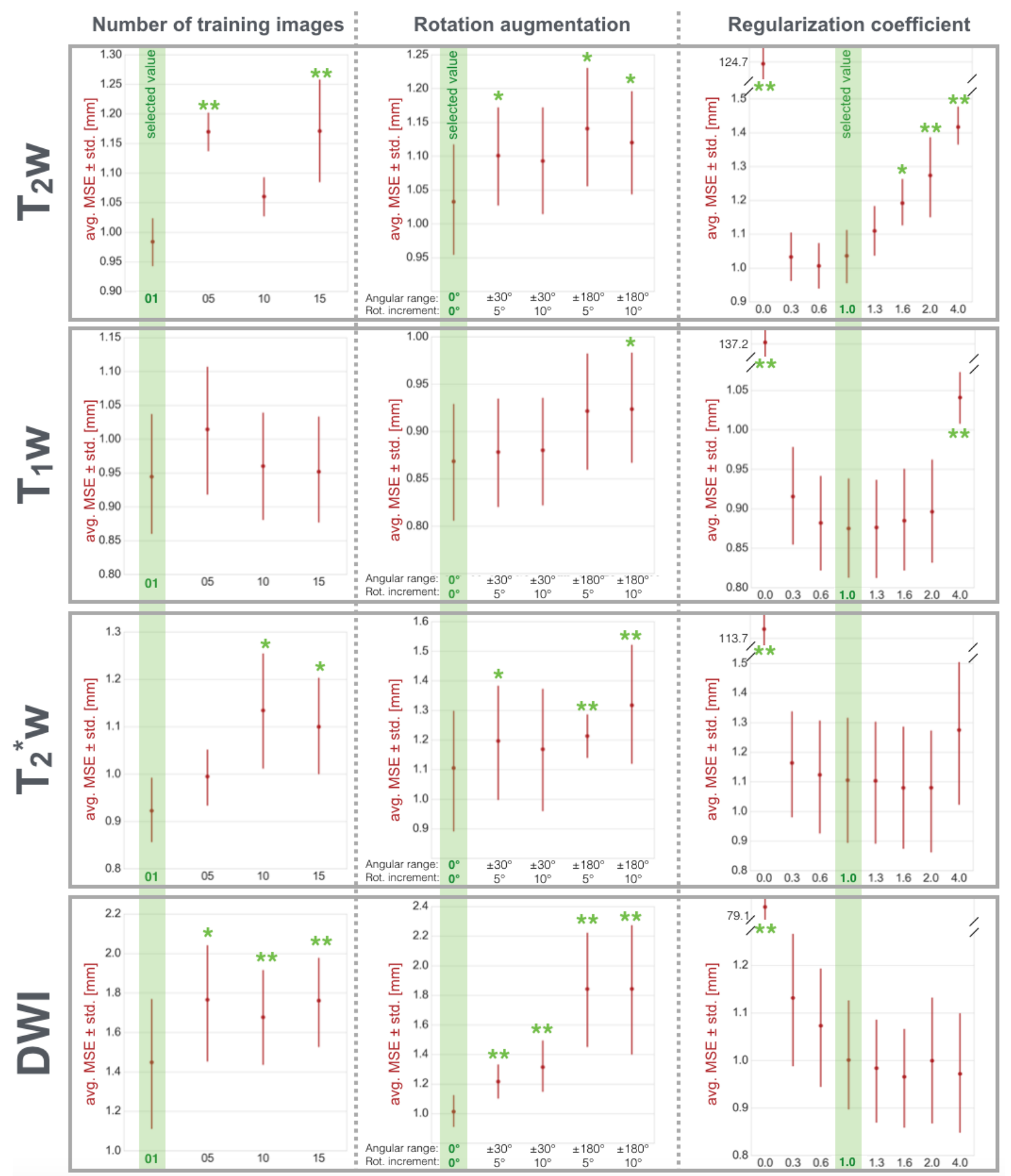

Figure 5. Hyperparameter Optimization. Three hyperparameters were investigated (from left to right): the number of training 3D images, the effect of rotation augmentation in training and the regularization coefficient. The optimization was done for the four contrasts (from top to bottom): $\mathrm{T}_{2} \mathrm{~W}, \mathrm{~T}_{1} \mathrm{~W}, \mathrm{~T}_{2}{ }^{*} \mathrm{~W}, \mathrm{DWI}$. Here, Avg. \pm Std. of the Mean Square Error (MSE) between the estimated centerline and the ground truth across 100 bootstrap iterations are plotted in red. For each hyperparameter optimization, $(*)$ indicates significant differences between the selected value (filled in green) and the other tested values, according to KruskalWallis H-tests (*: $\mathrm{p} \leq 0.05, * *$ : $\mathrm{p} \leq 0.01$ ). 


\subsection{OptiC SC centerline detection}

Figure 6 shows qualitative examples of SC centerline detection on healthy controls (HC) and patient volumes from the testing dataset. On both axial and sagittal views, SC centerlines were predicted closely to the ground-truth centerlines.
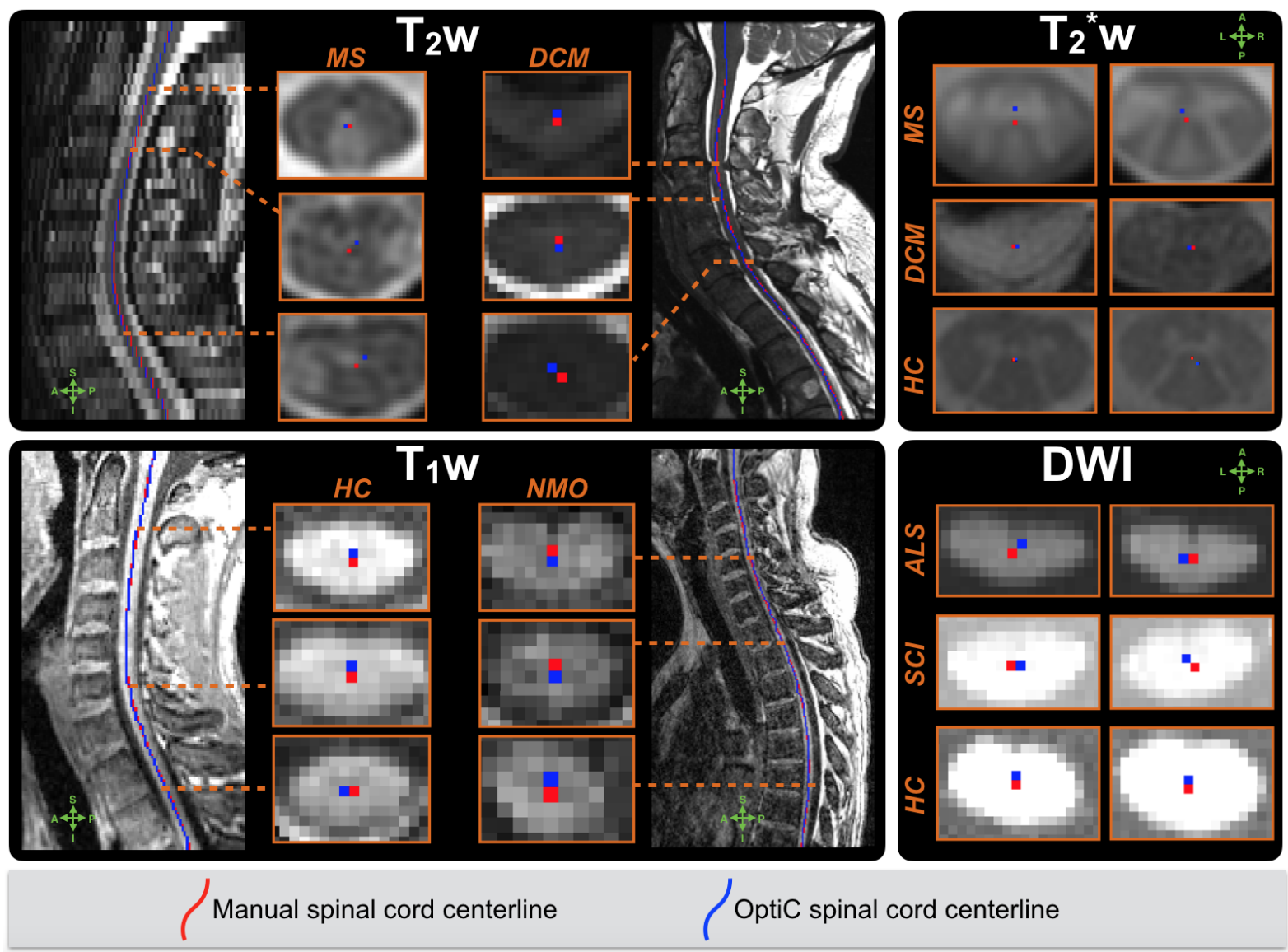

Figure 6. Examples of automatic spinal cord (SC) centerline detection on healthy controls (HC) and patients, on $\mathrm{T}_{2} \mathrm{~W}$ (top-left), $\mathrm{T}_{2}{ }^{*} \mathrm{w}$ (top-right), $\mathrm{T}_{1} \mathrm{~W}$ (bottom-left) and DWI (bottomright) images. This include a comparison between ground-truth (red) and automatic (blue) SC centerlines on axial and sagittal views. MS: multiple sclerosis; DCM: degenerative cervical myelopathy; NMO: neuromyelitis optica; SCI: traumatic spinal cord injury; ALS: amyotrophic lateral sclerosis.

OptiC performance was evaluated on testing datasets containing four contrasts, including $\mathrm{HC}$ and a large number of patients. OptiC was compared to the Hough method in Figure 7 in terms of localization rate and MSE. The localization rates were $98.77 \pm 4.49 \%$ for OptiC and $63.75 \pm 48.79 \%$ for Hough (averaged across contrasts $n=644$, Wilcoxon signedrank test p-value $<0.01$ ). The MSE results, for subjects with a detected cord (i.e. localization rate $>0 \%$ ), were $1.02 \pm 1.10 \mathrm{~mm}$ for OptiC and $9.95 \pm 13.76 \mathrm{~mm}$ for Hough (averaged across contrasts $n=485$, Wilcoxon signed-rank test $p$-value $<0.01$ ). Note that the Hough method performed adequately on $\mathrm{HC}$ but failed on most patients, whereas OptiC performed similarly well on both HC and patients. 


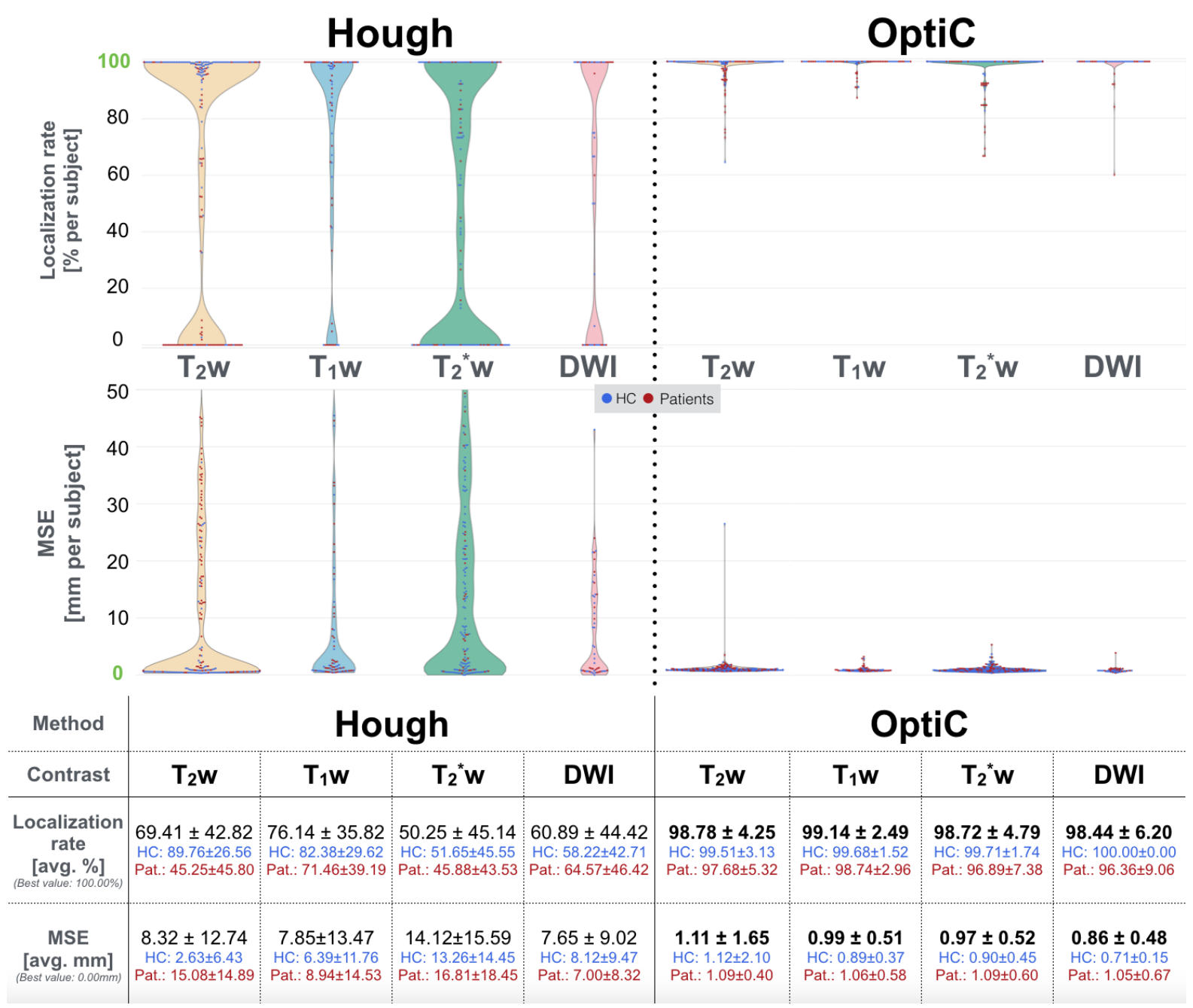

Figure 7. Results of the spinal cord (SC) localization in terms of localization rate (top) and Mean Square Error (MSE) between the ground-truth and predicted SC centerline (bottom). For each metric, the best value is indicated in green in the y-axis. Results are compared between the Hough method (De Leener et al., 2015) (left distribution plots) and the OptiC method (right distribution plots), where each point represents a healthy control (blue) or patient (red). Each method was evaluated on the 4 contrast datasets: $\mathrm{T}_{2} \mathrm{~W}, \mathrm{~T}_{1} \mathrm{~W}, \mathrm{~T}_{2}{ }^{*} \mathrm{~W}$ and $\mathrm{DWI}$ (from left to right). The table presents the average metrics per contrast for each method, and for the $\mathrm{HC}$ and the patient (Pat.) subgroups. 


\subsection{Pontomedullary junction detection}

Table 1 shows the results of the PMJ automatic detection on the $\mathrm{T}_{2} \mathrm{~W}$ and $\mathrm{T}_{1} \mathrm{~W}$ testing datasets. The proposed detection method provides good results in terms of recall $\left(\mathrm{T}_{2} \mathrm{~W}\right.$ : $84 \%$; $\mathrm{T}_{1} \mathrm{~W}$ : $\left.100 \%\right)$, with few FN detections, as well as high precision $\left(\mathrm{T}_{2} \mathrm{w}: 100 \% ; \mathrm{T}_{1} \mathrm{w}: 99 \%\right)$, with few FP detection. Moreover, the averaged signed distance error between the predicted and the true PMJ position along the (S-I) axis is in the range of $1 \mathrm{~cm}$ below the PMJ $\left(\mathrm{T}_{2} \mathrm{~W}:-11.41 \pm\right.$ $5.71 \mathrm{~mm} ; \mathrm{T}_{1} \mathrm{~W}:-5.75 \pm 10.29$ ), resulting in an adequate separation of brain and SC structures around the medulla oblongata (situated just caudal to the PMJ).

\begin{tabular}{|c|c|c|c|}
\hline & $\begin{array}{c}\text { Recall [\%] } \\
\text { (Best value: 100) }\end{array}$ & $\begin{array}{c}\text { Precision [\%] } \\
\text { (Best value: 100) }\end{array}$ & $\begin{array}{c}\text { Distance error [mm] } \\
\text { (Best value: 0.00) }\end{array}$ \\
\hline $\begin{array}{c}\mathbf{T}_{2} \mathbf{W} \\
\left(n=247, n_{T P}=111\right)\end{array}$ & 84 & 100 & $-11.41 \pm 5.71$ \\
\hline $\begin{array}{c}\mathbf{T}_{\mathbf{1}} \mathbf{W} \\
\left(n=80, n_{T P}=72\right)\end{array}$ & 100 & 99 & $-5.75 \pm 10.29$ \\
\hline
\end{tabular}

Table 1. Pontomedullary junction (PMJ) detection results on $\mathrm{T}_{2} \mathrm{~W}$ and $\mathrm{T}_{1} \mathrm{~W}$ testing datasets. Recall and precision evaluated the ability of the proposed method to automatically detect the presence of the PMJ in the MRI volumes, referred to as True Positive volumes (TP). The signed distance error measures the distance between the predicted and the true PMJ position along the (S-I) axis.

\subsection{Proof of concept: application to SC segmentation}

Automatic SC segmentation tasks remain a challenging problem on a variety of MRI data (De Leener et al., 2016), mainly due to the difficulty to robustly detect the SC. This localization is often the first step of automatic SC segmentation pipelines. Hence, as a proof of concept of OptiC's application, we used OptiC to initialize a publicly available SC segmentation method, PropSeg (De Leener et al., 2015). Figure 8 compared the results of PropSeg when initialized with the default method (called "Hough+PropSeg") and with the SC centerline provided by OptiC (called “OptiC+PropSeg”). Dice coefficients calculated 
between the predicted and the ground-truth segmentations were $0.87 \pm 0.14$ when using OptiC initialization versus $0.44 \pm 0.43$ when using the default initialization (averaged across contrasts $n=644$, Wilcoxon signed-rank test $p$-value $<0.01$ ). OptiC was particularly valuable in pathological cases with a Dice coefficient of $0.84 \pm 0.14$ versus $0.36 \pm 0.41$ when using the default initialization (averaged across contrasts $\mathrm{n}=259$, Wilcoxon signed-rank test $\mathrm{p}$ value $<0.01)$.

The "OptiC+PropSeg" results for $\mathrm{T}_{2} \mathrm{~W}$ and $\mathrm{T}_{1} \mathrm{w}$ datasets were obtained by using the centerline automatically cropped at the PMJ position. This additional post-processing slightly improved the Dice coefficient of segmentations on MRI volumes with PMJ sections: Dice coefficients were $0.89 \pm 0.13$ without cropping versus $0.90 \pm 0.11$ with PMJ cropping (averaged across contrasts $n=183$ ).

Moreover, the above results were obtained efficiently when using OptiC, $0.41 \pm 0.46$ seconds per 2D axial slice (including reading/writing tasks, on an iMac i7 4-cores $3.4 \mathrm{GHz}$ 8Gb RAM) versus $8.05 \pm 82.90$ seconds without OptiC initialization (averaged across contrasts $n=644$, Wilcoxon signed-rank test $p$-value $<0.01$ ). Note that, OptiC centerline extraction is not time consuming since it is achieved in $10.00 \pm 6.98$ milliseconds per $2 \mathrm{D}$ axial slice (averaged across contrasts $n=644$ ).

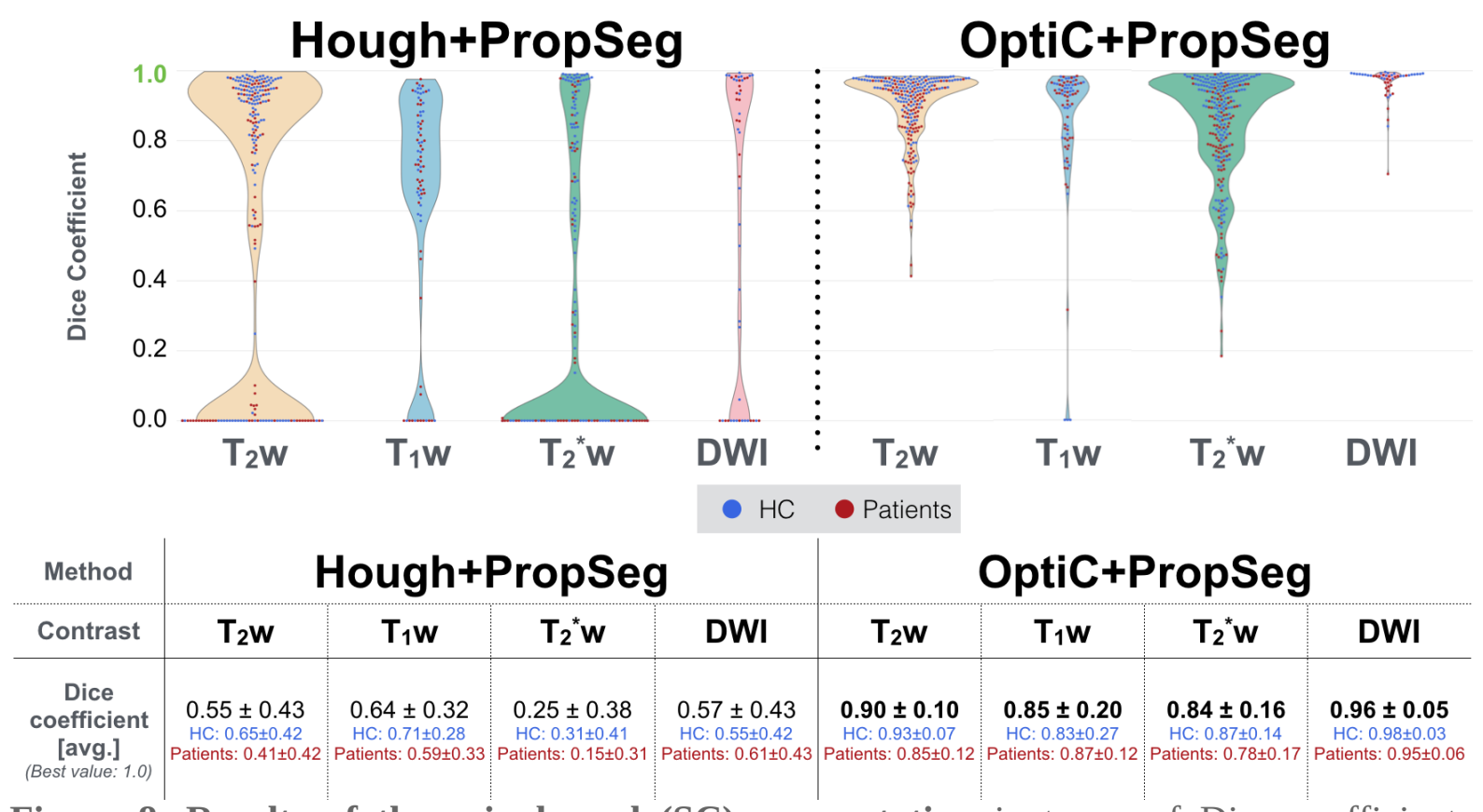

Figure 8. Results of the spinal cord (SC) segmentation in terms of Dice coefficients between the ground-truth and predicted SC segmentation. For each metric, the best value is indicated on green in the y-axis. Results are a comparison of PropSeg (De Leener et al., 2015) segmentations when centerline initialization is performed with the default method (left distribution plots) vs. the OptiC method (right distribution plots), where each point represents a healthy control (blue) or patient (red). Each method was evaluated on the 4 contrast datasets: $\mathrm{T}_{2} \mathrm{~W}, \mathrm{~T}_{1} \mathrm{~W}, \mathrm{~T}_{2}{ }^{*} \mathrm{~W}$ and $\mathrm{DWI}$ (from left to right). The table presents the averaged metrics per contrast for each method. 


\section{Discussion}

In this article, a new automatic and fast method to detect the center of the SC on MRI data was presented. The proposed method is based on a supervised machine learning detection method combined with a novel algorithm that efficiently provides the global solution of an optimization problem. Additionally, a new post-processing feature to detect and correct for the PMJ landmark allows the pipeline to handle datasets with a variety of image FOVs.

Key benefits of OptiC are (i) robustness to image artifacts (e.g. susceptibility, motion, ghosting, chemical shift, blurring, Gibbs) thanks to the sophisticated normalization HOG strategy (Dalal and Triggs, 2005), (ii) respect for anatomical cord continuity as a result of the regularization along the (S-I) axis, (iii) robustness of curve extraction ensured by the finding of the global minimum (iv) fast computation time by reason of a linear complexity with the number of voxels. Further efforts to extend our results might consider the use of more sophisticated object detection classifier, such as Convolutional Neural Networks (Long et al., 2015; Ronneberger et al., 2015), and/or other engineering features, such as SURF features (Bay et al., 2008/6).

The hyperparameter study shows that training the model with only one image provides good results. It suggests the possibility to fastly and easily create a new model if needed (e.g. adapted to a new contrast or to a specific pathology) without requiring a large amount of training 3D images. Rotation augmentation on the training data did not prove useful to detect the SC, even if HOG features are not inherently rotation invariant. This can probably be explained by the setting of a common image orientation in the preprocessing steps. The regularization coefficient optimization suggests that the solving of the optimization problem ( $\lambda^{\prime} \neq 0$ ) provides significant improvements compared to the SC localization map output by the classifier $\left(\lambda^{\prime}=0\right)$.

This study evaluated the performance of the OptiC method to automatically detect the SC on a variety of MRI data and to provide a reliable SC centerline. OptiC was validated on a large multi-center dataset with heterogeneous contrast, resolution, FOVs. Both accuracy and robustness of the method have been shown, even on challenging pathological cases with hyperintense lesions or cord deformations, such as in 3D images from patients with MS $(n=79)$ or DCM ( $n=63)$ : the averaged MSE from patients was $1.08 \mathrm{~mm}$ vs. $0.97 \mathrm{~mm}$ from controls. The robustness of the proposed method provides a proof-of-concept to allow more robust automatic analysis on pathological cases with clinical applications.

The automatic detection of the PMJ allows the extraction of a SC centerline restricted to SC regions even on MRI volumes that include the brain. Other anatomical structures such as the top of $\mathrm{C} 1$ vertebra or the bottom of the medulla oblongata might also have been appropriate to separate the brain and SC regions, but these structures are much more 
challenging to label consistently and to detect automatically. The straightening of the SC is a necessary step to robustly detect the PMJ, however, the computation of the warping fields is time consuming. Validation results demonstrate the precision of the PMJ detection method (low rate of FP detections) and its accuracy ( $<1 \mathrm{~cm}$ below the PMJ on average). Note that, the suggested separation position is situated on average within the medulla oblongata, below the other (more superior) brain regions. Notably, its benefit for SC segmentation tasks was shown by slightly improving the Dice coefficient of the segmentation. Therefore, delineating the SC below the medulla oblongata would have benefits when integrated on fully automatic pipelines.

This paper shows the benefits of the OptiC centerline to initialize a state-of-the-art automatic SC segmentation technique (De Leener et al., 2015). Automatic detection of the SC has also recently been proposed to improve B0 field correction in targeted optimization of shim currents (Johanna Vannesjo et al., 2017; Topfer et al., 2016) to adapt the shimming to the exact position of the SC in $\mathrm{T}_{2}{ }^{*}-\mathrm{w}$ images. Other applications, such as template registration or shape analysis, could also benefit from this method. 


\section{Conclusion}

SC centerline detection is a key step to initialize fully automatic pipelines in various applications such as SC segmentation, shape analysis, template registration or B0 field correction. In this paper, a fully automated method for localizing the SC from MRI data was presented, extensively validated on a large multi-center heterogeneously acquired datasets with a large variety of pathologies and successfully compared with a state-of-the-art SC localization technique. The proposed SC centerline detection method is freely available as part of the Spinal Cord Toolbox: http://sourceforge.net/projects/spinalcordtoolbox/. 


\section{Acknowledgements:}

- We would like to acknowledge all members from the NeuroPoly laboratory for useful and constructive discussions.

- Acknowledged for sharing data: Manuel Taso (University of Aix-Marseille); Julius Dewald and Haleh Karbasforoushan (Northwestern University); Seth Smith, Bailey Lyttle, Ben Conrad and Bennett Landman (Vanderbilt University); Barry Bedell (Biospective Inc.); Seok Woo Kim and Jisun Song (Hallym University); Emil Ljungberg (University of British Columbia); Eric Klawiter (Massachusetts General Hospital); Maryam Seif and Patrick Freund (University Hospital Zurich); PierreFrançois Pradat and Habib Benali (Pitié-Salpêtrière Hospital); Pierre Rainville (Canadian Institutes of Health Research); Rohit Bakshi, Subhash Tummala and Shahamat Tauhid (Brigham and Women's Hospital, Harvard Medical School); Gilles Edan, Anne Kerbrat and Elise Bannier (University Hospital of Rennes); Pierre Labauge (University Hospital of Montpellier); Ferran Prados and Claudia Gandini Wheeler-Kingshott (University College London); Yaou Liu and Zhuoqiong Ren (Xuanwu Hospital); Tom Lillicrap (University of Newcastle).

- This work was supported by: Canada Research Chair in Quantitative Magnetic Resonance Imaging (JCA), the Canadian Institute of Health Research [CIHR FDN143263], the Canada Foundation for Innovation [32454], the Fonds de Recherche du Québec - Santé [28826], the Fonds de Recherche du Québec - Nature et Technologies [2015-PR-182754, 2017-200599], the Natural Sciences and Engineering Research Council of Canada [435897-2013], the Quebec BioImaging Network, the Program "Investissements d'Avenir" operated by the French National Research Agency [ANR11-INBS-0006], the LABEX PRIMES [ANR-11-LABX-0063] of Université de Lyon, "Investissements d'Avenir" [ANR-11-IDEX-0007], the National Multiple Sclerosis Society NMSS RG-1501-02840 (SAS), the Centre National de la Recherche Scientifique (CNRS), the "Fondation A*midex-Investissements d'Avenir" and the "Fondation Aix-MArseille Université”, NIH/NINDS R21 NS087465-01 (SAS), NIH/NEI R01 EY023240 (SAS), DoD W81XWH-13-0073 (SAS), Grant MOP13034. 


\section{References}

Bay, H., Ess, A., Tuytelaars, T., Van Gool, L., 2008/6. Speeded-Up Robust Features (SURF). Comput. Vis. Image Underst. 110, 346-359.

Bradski, G., Kaehler, A., 2008. Learning OpenCV: Computer Vision with the OpenCV Library. “O’Reilly Media, Inc.”

Carbonell-Caballero, J., Manjón, J.V., Martí-Bonmatí, L., Olalla, J.R., Casanova, B., de la IglesiaVayá, M., Coret, F., Robles, M., 2006. Accurate quantification methods to evaluate cervical cord atrophy in multiple sclerosis patients. MAGMA 19, 237-246.

Cawley, N., Tur, C., Prados, F., Plantone, D., Kearney, H., Abdel-Aziz, K., Ourselin, S., WheelerKingshott, C.A.G., Miller, D.H., Thompson, A.J., Ciccarelli, O., 2017. Spinal cord atrophy as a primary outcome measure in phase II trials of progressive multiple sclerosis. Mult. Scler. 1352458517709954.

Chen, M., Carass, A., Oh, J., Nair, G., Pham, D.L., Reich, D.S., Prince, J.L., 2013. Automatic magnetic resonance spinal cord segmentation with topology constraints for variable fields of view. Neuroimage 83, 1051-1062.

Cohen-Adad, J., El Mendili, M.-M., Lehéricy, S., Pradat, P.-F., Blancho, S., Rossignol, S., Benali, H., 2011. Demyelination and degeneration in the injured human spinal cord detected with diffusion and magnetization transfer MRI. Neuroimage 55, 1024-1033.

Cohen-Adad, J., El Mendili, M.-M., Morizot-Koutlidis, R., Lehéricy, S., Meininger, V., Blancho, S., Rossignol, S., Benali, H., Pradat, P.-F., 2013. Involvement of spinal sensory pathway in ALS and specificity of cord atrophy to lower motor neuron degeneration. Amyotroph. Lateral Scler. Frontotemporal Degener. 14, 30-38.

Dalal, N., Triggs, B., 2005. Histograms of oriented gradients for human detection, in: 2005 IEEE Computer Society Conference on Computer Vision and Pattern Recognition (CVPR'05). pp. 886-893 vol. 1.

De Leener, B., Cohen-Adad, J., Kadoury, S., 2015. Automatic Segmentation of the Spinal Cord and Spinal Canal Coupled With Vertebral Labeling. IEEE Trans. Med. Imaging 34, 1705-1718.

De Leener, B., Kadoury, S., Cohen-Adad, J., 2014. Robust, accurate and fast automatic segmentation of the spinal cord. Neuroimage 98, 528-536.

De Leener, B., Lévy, S., Dupont, S.M., Fonov, V.S., Stikov, N., Louis Collins, D., Callot, V., CohenAdad, J., 2017a. SCT: Spinal Cord Toolbox, an open-source software for processing spinal cord MRI data. Neuroimage 145, 24-43.

De Leener, B., Mangeat, G., Dupont, S., Martin, A.R., Callot, V., Stikov, N., Fehlings, M.G., CohenAdad, J., 2017b. Topologically preserving straightening of spinal cord MRI. J. Magn. Reson. Imaging. doi:10.1002/jmri.25622

De Leener, B., Taso, M., Cohen-Adad, J., Callot, V., 2016. Segmentation of the human spinal cord. MAGMA 29, 125-153.

Dice, L.R., 1945. Measures of the Amount of Ecologic Association Between Species. Ecology 26, 297-302.

Dupont, S.M., De Leener, B., Taso, M., Le Troter, A., Nadeau, S., Stikov, N., Callot, V., Cohen-Adad, J., 2017. Fully-integrated framework for the segmentation and registration of the spinal cord white and gray matter. Neuroimage 150, 358-372.

Ellingson, B.M., Ulmer, J.L., Schmit, B.D., 2008. Morphology and morphometry of human chronic spinal cord injury using diffusion tensor imaging and fuzzy logic. Ann. Biomed. Eng. 36, 224 236.

Felzenszwalb, P., Huttenlocher, D., 2004. Distance transforms of sampled functions. Cornell University.

Fradet, L., Arnoux, P.-J., Ranjeva, J.-P., Petit, Y., Callot, V., 2014. Morphometrics of the entire human spinal cord and spinal canal measured from in vivo high-resolution anatomical magnetic resonance imaging. Spine 39, E262-9.

Grabher, P., Mohammadi, S., David, G., Freund, P., 2017. Neurodegeneration in the spinal ventral horn prior to motor impairment in cervical spondylotic myelopathy. J. Neurotrauma. doi:10.1089/neu.2017.4980

Horsfield, M.A., Sala, S., Neema, M., Absinta, M., Bakshi, A., Sormani, M.P., Rocca, M.A., Bakshi, R., Filippi, M., 2010. Rapid semi-automatic segmentation of the spinal cord from magnetic resonance images: application in multiple sclerosis. Neuroimage 50, 446-455. 
Jenkinson, M., Beckmann, C.F., Behrens, T.E.J., Woolrich, M.W., Smith, S.M., 2012. FSL. Neuroimage 62, 782-790.

Johanna Vannesjo, S., Shi, Y., Tracey, I., Miller, K.L., Clare, S., 2017. Slice-wise rst-order shimming of the human spinal cord at 7T, in: Proceedings of the 25th Annual Meeting of ISMRM. p. 5210.

Koh, J., Kim, T., Chaudhary, V., Dhillon, G., 2010. Automatic segmentation of the spinal cord and the dural sac in lumbar MR images using gradient vector flow field. Conf. Proc. IEEE Eng. Med. Biol. Soc. 2010, 3117-3120.

Long, J., Shelhamer, E., Darrell, T., 2015. Fully convolutional networks for semantic segmentation, in: Proceedings of the IEEE Conference on Computer Vision and Pattern Recognition. pp. 34313440.

Martin, A.R., De Leener, B., Cohen-Adad, J., Cadotte, D.W., Kalsi-Ryan, S., Lange, S.F., Tetreault, L., Nouri, A., Crawley, A., Mikulis, D.J., Ginsberg, H., Fehlings, M.G., 2017a. A Novel MRI Biomarker of Spinal Cord White Matter Injury: T2*-Weighted White Matter to Gray Matter Signal Intensity Ratio. AJNR Am. J. Neuroradiol. doi:10.3174/ajnr.A5162

Martin, A.R., De Leener, B., Cohen-Adad, J., Cadotte, D.W., Kalsi-Ryan, S., Lange, S.F., Tetreault, L., Nouri, A., Crawley, A., Mikulis, D.J., Others, 2017b. Clinically feasible microstructural MRI to quantify cervical spinal cord tissue injury using DTI, MT, and T2*-weighted imaging: assessment of normative data and reliability. AJNR Am. J. Neuroradiol.

Meijster, A., J B T, Hesselink, W.H., 2002. A General Algorithm for Computing Distance Transforms in Linear Time, in: Goutsias, J., Vincent, L., Bloomberg, D.S. (Eds.), Mathematical Morphology and Its Applications to Image and Signal Processing, Computational Imaging and Vision. Springer US, pp. 331-340.

Nakamura, M., Miyazawa, I., Fujihara, K., Nakashima, I., Misu, T., Watanabe, S., Takahashi, T., Itoyama, Y., 2008. Preferential spinal central gray matter involvement in neuromyelitis optica. J. Neurol. 255, 163-170.

Papinutto, N., Schlaeger, R., Panara, V., Zhu, A.H., Caverzasi, E., Stern, W.A., Hauser, S.L., Henry, R.G., 2015. Age, gender and normalization covariates for spinal cord gray matter and total crosssectional areas at cervical and thoracic levels: A 2D phase sensitive inversion recovery imaging study. PLoS One 10, e0118576.

Pezold, S., Fundana, K., Amann, M., Andelova, M., Pfister, A., Sprenger, T., Cattin, P.C., 2015. Automatic Segmentation of the Spinal Cord Using Continuous Max Flow with Cross-sectional Similarity Prior and Tubularity Features, in: Yao, J., Glocker, B., Klinder, T., Li, S. (Eds.), Recent Advances in Computational Methods and Clinical Applications for Spine Imaging, Lecture Notes in Computational Vision and Biomechanics. Springer International Publishing, pp. 107-118.

Prados, F., Ashburner, J., Blaiotta, C., Brosch, T., Carballido-Gamio, J., Cardoso, M.J., Conrad, B.N., Datta, E., Dávid, G., Leener, B.D., Dupont, S.M., Freund, P., Wheeler-Kingshott, C.A.M.G., Grussu, F., Henry, R., Landman, B.A., Ljungberg, E., Lyttle, B., Ourselin, S., Papinutto, N., Saporito, S., Schlaeger, R., Smith, S.A., Summers, P., Tam, R., Yiannakas, M.C., Zhu, A., CohenAdad, J., 2017. Spinal cord grey matter segmentation challenge. Neuroimage 152, 312-329.

Prados, F., Cardoso, M.J., Yiannakas, M.C., Hoy, L.R., Tebaldi, E., Kearney, H., Liechti, M.D., Miller, D.H., Ciccarelli, O., Wheeler-Kingshott, C.A.M.G., Ourselin, S., 2016. Fully automated grey and white matter spinal cord segmentation. Sci. Rep. 6, 36151.

Ronneberger, O., Fischer, P., Brox, T., 2015. U-Net: Convolutional Networks for Biomedical Image Segmentation, in: Navab, N., Hornegger, J., Wells, W.M., Frangi, A.F. (Eds.), Medical Image Computing and Computer-Assisted Intervention - MICCAI 2015, Lecture Notes in Computer Science. Presented at the International Conference on Medical Image Computing and ComputerAssisted Intervention, Springer International Publishing, pp. 234-241.

Stroman, P.W., Figley, C.R., Cahill, C.M., 2008. Spatial normalization, bulk motion correction and coregistration for functional magnetic resonance imaging of the human cervical spinal cord and brainstem. Magn. Reson. Imaging 26, 809-814.

Stroman, P.W., Wheeler-Kingshott, C., Bacon, M., Schwab, J.M., Bosma, R., Brooks, J., Cadotte, D., Carlstedt, T., Ciccarelli, O., Cohen-Adad, J., Curt, A., Evangelou, N., Fehlings, M.G., Filippi, M., Kelley, B.J., Kollias, S., Mackay, A., Porro, C.A., Smith, S., Strittmatter, S.M., Summers, P., Tracey, I., 2014. The current state-of-the-art of spinal cord imaging: Methods. Neuroimage 84, $1070-1081$.

Topfer, R., Starewicz, P., Lo, K.-M., Metzemaekers, K., Jette, D., Hetherington, H.P., Stikov, N., Cohen-Adad, J., 2016. A 24-channel shim array for the human spinal cord: Design, evaluation, and application. Magn. Reson. Med. 76, 1604-1611.

Yiannakas, M.C., Mustafa, A.M., De Leener, B., Kearney, H., Tur, C., Altmann, D.R., De Angelis, F., 
Plantone, D., Ciccarelli, O., Miller, D.H., Cohen-Adad, J., Gandini Wheeler-Kingshott, C.A.M., 2016. Fully automated segmentation of the cervical cord from T1-weighted MRI using PropSeg: Application to multiple sclerosis. Neuroimage Clin 10, 71-77. 